\title{
Zero-energy peak of the density of states and localization properties of a one-dimensional Frenkel exciton: Off-diagonal disorder
}

\author{
G. G. Kozlov and V. A. Malyshev \\ All-Russian Research Center “Vavilov State Optical Institute,”' Birzhevaya Liniya 12, 199034 Saint Petersburg, Russia \\ F. Domínguez-Adame \\ GISC, Departamento de Física de Materiales, Universidad Complutense, E-28040 Madrid, Spain
}

\section{A. Rodríguez}

GISC, Departamento de Matemática Aplicada y Estadística, Universidad Politécnica, E-28040 Madrid, Spain

(Received 24 October 1997; revised manuscript received 27 January 1998)

\begin{abstract}
We study a one-dimensional Frenkel Hamiltonian with off-diagonal disorder, focusing our attention on the physical nature of the zero-energy peak of the density of states. The character of excitonic states (localized or delocalized) is also examined in the vicinity of this peak by means of the inverse participation ratio. It is shown that the state being responsible for the peak is localized. A detailed comparison of the nearest-neighbor approach with the long-range dipole-dipole coupling is performed. [S0163-1829(98)03632-7]
\end{abstract}

\section{INTRODUCTION}

Since the pioneering works of Anderson, ${ }^{1}$ and Mott and Twose, ${ }^{2}$ electronic and transport properties of randomly disordered systems have been the subject of long-lasting interest both from fundamental and applied viewpoints. ${ }^{1-5}$ Onedimensional (1D) systems are frequently considered because they turn out to be simpler than those in three dimensions. ${ }^{3}$ Originally, Mott and Twose ${ }^{2}$ conjectured that all states are localized in 1D systems, for any degree of disorder. Afterwards, a great deal of work has been devoted to examine the Mott-Twose conjecture (see, for instance, Ref. 6). However, it is well-known that electron delocalization appears in 1D random systems with short-range correlations. ${ }^{7,8}$

Two decades ago, Theodorou and Cohen established that the density of states (DOS) of a 1D tight-binding Hamiltonian with nearest-neighbor (NN) interactions and random off-diagonal elements presents a singularity at the center of the band. ${ }^{9}$ These authors used an analytical approach based on previous results obtained by Dyson ${ }^{10}$ for disordered linear chains of harmonic oscillators. In Ref. 9, it was also stated that the corresponding state is delocalized as the localization length was found to be infinite. Adding some amount of diagonal disorder in the presence of off-diagonal randomness makes all states localized. ${ }^{11}$ Remarkably, the first calculations on 1D tight-binding Hamiltonians with only diagonal disorder did not reveal any singularity either in the DOS or in the localization length. ${ }^{12,13}$ Further, a very weak anomaly (a peak but not a singularity) in both properties mentioned above was found both numerically ${ }^{14}$ and analytically. ${ }^{15,16}$

Recently, Fidder et al. have found by numerical diagonalization of the 1D Frenkel Hamiltonian with off-diagonal disorder that, notwithstanding the singularity of the DOS, the corresponding state is localized if one includes the longrange (LR) interactions due to dipolar coupling between different sites. ${ }^{17}$ This finding seems to be in contradiction with the point of view raised in Ref. 9 suggesting that the state corresponding to the singularity of the DOS is delocalized.
In this paper, we examine in detail the conclusions of Refs. 9 and 17 . We address this issue by considering a 1D Frenkel Hamiltonian with off-diagonal disorder with NN interactions and compare the results with those obtained when LR interactions are taken into account. The rest of the paper is organized as follows. In Sec. II, the 1D Frenkel Hamiltonian with $\mathrm{NN}$ interactions is analyzed. We present arguments against those raised in Ref. 9, namely, that the zero-energy state is localized, even in the NN problem. This conclusion, based on analytical considerations, is then confirmed by direct diagonalization of the Hamiltonian. The detailed study of the 1D Frenkel Hamiltonian with LR interactions is presented in Sec. III. Section IV deals with the numerical simulations confirming the analytical results. Using numerical diagonalization of a Frenkel Hamiltonian with LR interactions, we calculate both the DOS and the inverse participation ratio, to be defined below, and study new features of these magnitudes with respect to the NN approach. Section V concludes with some comments regarding the results we have obtained.

\section{IS THE ZERO-ENERGY STATE DELOCALIZED?}

In this section we briefly review the arguments of Ref. 9 leading to the conclusion that the state at the center of the band is delocalized. We present further arguments suggesting the opposite point of view and, what is most important, numerical calculations confirm our statement. Let us consider a tight-binding Hamiltonian with only $\mathrm{NN}$ interactions,

$$
H=\sum_{n} U_{n, n+1}(|n\rangle\langle n+1|+| n+1\rangle\langle n|),
$$

where the NN interactions $\left\{U_{n, n+1}\right\}$ are assumed to be $\delta$ correlated and similarly distributed stochastic variables. The state vector $|n\rangle$ represents an excitation at site $n$. All site energies are set to zero since no diagonal disorder is included. The eigenvalue problem of the NN model reads 


$$
U_{n, n+1} a_{n+1}+U_{n, n-1} a_{n-1}=E a_{n},
$$

where the set $\left\{a_{n}\right\}$ represents the real eigenvector corresponding to the eigenenergy $E$. For zero energy Eq. (2) gives the recurrence relation $a_{n+1}=-\left(U_{n, n-1} / U_{n, n+1}\right) a_{n-1}$. Using this relation one can find

$$
a_{2 n+1}=\left(-\frac{U_{2 n, 2 n-1}}{U_{2 n, 2 n+1}}\right)\left(-\frac{U_{2 n-2,2 n-3}}{U_{2 n-2,2 n-1}}\right) \ldots\left(-\frac{U_{2,1}}{U_{2,3}}\right) a_{1} .
$$

The amplitudes at even positions vanish. The eigenvector (3) represents the zero-energy state for a chain with odd number of sites. Defining the localization length at the center of the band $L(E=0)$ by the expression

$$
\frac{1}{L(E=0)}=-\lim _{n \rightarrow \infty} \frac{1}{2 n} \ln \left|\frac{a_{2 n+1}}{a_{1}}\right|,
$$

and applying the central-limit theorem, the authors of Ref. 9 obtained $1 / L(E=0)=0$. From this result they concluded that the state at center of the band was extended.

The definition of the localization length (4) is based on an unconditional assumption of the so-called exponential localization. Indeed, in such a case one would have typically $a_{2 n+1} \sim \exp [-(2 n+1) / L]$. Certainly, the definition (4) cannot discern between a weaker than exponentially localized state (where the amplitude $a_{2 n+1}$ decreases or increases with $n$ slower than an exponential) and an extended state (where $a_{2 n+1} \sim 1 / \sqrt{N}$ with $N \rightarrow \infty$ being the number of sites in the chain). In such a case, the mean extension of the eigenfunctions or the inverse participation ratio are the more adequate quantities for learning the character of the state.

The results presented below show that the problem we are discussing just belongs to those that cannot be adequately analyzed from the assumptions leading to Eq. (4). First, let us write the $\mathrm{NN}$ interactions in the form $U_{n, n+1}=U_{0}(1$ $\left.+\xi_{n, n+1}\right)$, where $\xi_{n, n+1}$ are Gaussian distributed stochastic variables with variance $\xi_{0}^{2} \ll 1$. Then, it is easy to calculate the probability distribution of $\xi_{2 n+1} \equiv \ln \left|a_{2 n+1} / a_{1}\right|$,

$$
g\left(\xi_{2 n+1}\right)=\frac{1}{\sqrt{4 \pi n} \xi_{0}} \exp \left(-\frac{\xi_{2 n+1}^{2}}{4 \xi_{0}^{2} n}\right) .
$$

From this, the authors of Ref. 18 claimed that typically $\left|a_{2 n+1} / a_{1}\right| \sim \exp \left( \pm 2 \xi_{0} \sqrt{n}\right)$. If so, one should conclude that the zero-energy state is localized rather than extended, in contradiction with the statement of Ref. 9.

Further, using Eq. (5) we can calculate the probability distribution of $\chi_{2 n+1} \equiv\left|a_{2 n+1} / a_{1}\right|$,

$$
f\left(\chi_{2 n+1}\right)=\frac{1}{\sqrt{4 \pi n} \xi_{0} \chi_{2 n+1}} \exp \left(-\frac{\ln ^{2} \chi_{2 n+1}}{4 \xi_{0}^{2} n}\right) .
$$

This function has a sharp peak at $\chi_{\max }=\exp \left(-2 \xi_{0}^{2} n\right)$ and a very broad tail for large $\chi_{2 n+1}$ such that $\left\langle\chi_{2 n+1}\right\rangle$ $=\int \chi f(\chi) d \chi=\exp \left(\xi_{0}^{2} n\right)$. Thus, it is rather difficult to make a definite conclusion from Eq. (6) concerning a typical dependence of $\left|a_{2 n+1} / a_{1}\right|$ on $n$. Nevertheless, the fact that $f(0)$ $=0$ certainly indicates the zero probability to obtain an extended state. Below, we confirm this observation by numerical simulations.

\section{FRENKEL HAMILTONIAN}

We will be also interested in studying both the DOS and the degree of localization of states of a 1D tight-binding Hamiltonian including all (LR) interactions, beyond the NN interactions. According to this, we then introduce the complete Hamiltonian

$$
\mathcal{H}=\sum_{\substack{m, n=1 \\ m \neq n}}^{N} U_{m n}|m\rangle\langle n|,
$$

in which summation is performed now over all pairs of sites. For definiteness, it is assumed hereafter that excitations described by the presented Hamiltonian correspond to Frenkel excitons. Furthermore, the $U_{m n}$ is assumed to be of dipoledipole nature. We restrict ourselves to the case in which all transition dipole moments have the same magnitude and direction. Thus, one can take $U_{m n}=-U /\left|\xi_{m}-\xi_{n}\right|^{3}$, where $-U(U>0)$ is the dipole-dipole coupling of nearest neighbors in the periodic lattice, i.e., at $\xi_{m}-\xi_{m+1}=1$ (we chose here the negative sign of NN coupling as it takes place, for example, in $J$ aggregates ${ }^{17}$ ), and $\xi_{m}=m+\delta_{m}$ with $\delta_{m}$ being stochastic variables assumed to be distributed around the regular sequence according to the Gaussian law with variance $\sigma^{2}$,

$$
\mathcal{P}\left(\delta_{m}\right)=\left(\frac{1}{2 \pi \sigma^{2}}\right)^{1 / 2} \exp \left(-\frac{\delta_{m}^{2}}{2 \sigma^{2}}\right) .
$$

\section{A. The exciton spectrum and the DOS in the absence of disorder}

Before any discussions of the effects resulted from localization, it is useful to recall the peculiar features of the 1Dexciton spectrum and of the DOS in the absence of disorder $\left(\delta_{m}=0\right)$. Then the Hamiltonian (7) can be approximately diagonalized (with accuracy of the order of $N^{-1}$ ) by introducing the excitonic basis ${ }^{19}$

$$
|k\rangle=\left(\frac{2}{N+1}\right)^{1 / 2} \sum_{n=1}^{N} \sin \left(\frac{\pi k n}{N+1}\right)|n\rangle .
$$

The state vector $|k\rangle$ represents an exciton in the $k$ th state. Substituting Eq. (9) into Eq. (7) one obtains ${ }^{19}$

$$
\begin{gathered}
\mathcal{H}=\sum_{k=1}^{N} E_{k}|k\rangle\langle k|, \\
E_{k}=-2 U \sum_{n=1}^{N} \frac{1}{n^{3}} \cos \left(\frac{\pi k n}{N+1}\right)+O\left(N^{-1}\right) .
\end{gathered}
$$

Equation (10b) generalizes the corresponding expression of the NN approximation $[n=1$ term in Eq. (10b)] to the case of including all (LR) interactions. We are especially interested in the behavior of the spectrum and of the DOS in the vicinity of extreme points, $K \equiv \pi k /(N+1)=0$ and $K=\pi$, as well as at the center of the band, $k=(N+1) / 2(N$ taken to be odd). To do that, we exploit the following equation: ${ }^{20}$

$$
\sum_{n=1}^{\infty} \frac{\cos K n}{n}=-\ln \left(2 \sin \frac{K}{2}\right)
$$


and the fact that one can extend the sum in Eq. (10b) up to infinity because $n^{-3}$ decreases with $n$ fast enough. Then, by integrating Eq. (11) twice with respect to $K$, the sum in Eq. (10b) can be cast into the form ${ }^{19}$

$$
\begin{gathered}
E_{k}=-2 U \zeta(3)+U\left(\frac{3}{2}-\ln K\right) K^{2}, \quad K \ll 1, \\
E_{k}=\frac{3}{2} U \zeta(3)-U \ln 2(K-\pi)^{2}, \quad K-\pi \ll 1,
\end{gathered}
$$

where $\zeta(3)=\sum_{n=1}^{\infty} n^{-3} \approx 1.202$. The corresponding formulas within the NN approximation are $E_{k}=-2 U+U K^{2}$ if $K \ll 1$ and $E_{k}=2 U-U(K-\pi)^{2}$ if $K-\pi \ll 1$. Thus, one can conclude that LR interactions affect the position of both the bottom and the top of the band as well as the DOS of 1D excitons. As we can see, the bottom and top of the band change, respectively, shift from $-2 U$ to $-2 U \zeta(3) \approx$ $-2.404 U$ and from $2 U$ to $(3 / 2) U \zeta(3) \approx 1.803 U$. The DOS decreases approximately by the factor $\sqrt{\ln |E|}$ in the vicinity of the bottom of the band and, on the contrary, grows by the factor $1 / \ln 2$ close the top.

Finally, we would like to comment on the energy of the central exciton band state, with $k=(N+1) / 2$. In the $\mathrm{NN}$ model, one finds that $E_{(N+1) / 2}=0$, while with including all dipolar couplings, this energy is shifted to

$$
E_{(N+1) / 2}=-2 U \sum_{n=1}^{N} \frac{1}{n^{3}} \cos \left(\frac{\pi n}{2}\right) \approx 0.225 U .
$$

The DOS in the vicinity of the band center does not change noticeably as compared to the NN model.

\section{B. Motional narrowing effect}

In the presence of disorder, the Hamiltonian of the system can be written as a sum of two parts: the unperturbed one (10) and a term produced by the fluctuations of $U_{m n}$,

$$
\begin{gathered}
\mathcal{H}=\sum_{k=1}^{N} E_{k}|k\rangle\left\langle k\left|+\sum_{k, k^{\prime}=1}^{N} \Delta_{k k^{\prime}}\right| k\right\rangle\left\langle k^{\prime}\right|, \\
\Delta_{k k^{\prime}}=\frac{2}{N+1} \sum_{m, n=1}^{N} \delta U_{m n} \sin \left(\frac{\pi k n}{N+1}\right) \sin \left(\frac{\pi k^{\prime} m}{N+1}\right),
\end{gathered}
$$

where $\delta U_{m n}=U_{m n}-\bar{U}$, where $\bar{U}$ means averaging over the probability distribution (8). Here $\Delta_{k k^{\prime}}$ have diagonal and offdiagonal parts. The former is responsible only for the inhomogeneous broadening of excitonic levels, while the latter couples the excitonic modes and, therefore, causes the localization effects.

The $\Delta_{k k^{\prime}}$ undergo fluctuations because $\delta U_{m n}$ fluctuate. Assuming NN coupling and that $\delta U_{m n}$ fluctuations are small in some sense (see below), we can find the $\Delta_{k k^{\prime}}$ distribution in an analytical form. This also helps us to comment on the results of numerical simulations that we discuss later in Sec. IV.

In order to achieve the task, we use the definition

$$
\begin{aligned}
\mathcal{P}\left(\Delta_{k k^{\prime}}\right)= & \left\langle\delta \left\{\Delta_{k k^{\prime}}-\frac{2}{N+1} \sum_{n=1}^{N-1} \delta U_{n, n+1}\right.\right. \\
& \times\left[\sin \left(\frac{\pi k n}{N+1}\right) \sin \left(\frac{\pi k^{\prime}(n+1)}{N+1}\right)\right. \\
& \left.\left.\left.+\sin \left(\frac{\pi k^{\prime} n}{N+1}\right) \sin \left(\frac{\pi k(n+1)}{N+1}\right)\right]\right\}\right\rangle .
\end{aligned}
$$

Here, angular brackets indicate the average of the $\delta$ function over the fluctuations of NN distances. They obey a Gaussian distribution law like Eq. (8) but replace $\sigma^{2}$ by $\sigma_{N N}^{2}=2 \sigma^{2}$. We omit the details of tedious but straightforward trigonometric calculations and only quote the final results.

It can be easily shown that the sum in Eq. (15) is exactly equal to zero if $k+k^{\prime}=N+1$. Particularly, this means that $\Delta_{k k^{\prime}}=0$ for $k=k^{\prime}=(N+1) / 2$ when $N$ is taken to be odd, i.e., the first-order correction to the central energy is exactly equal to zero and does not fluctuate. Fluctuations of the other $\Delta_{k k^{\prime}}$ are distributed according to the Gaussian function with variances of the diagonal and off-diagonal elements distribution, $\sigma_{d}^{2}(k)$ and $\sigma_{n d}^{2}\left(k, k^{\prime}\right)$, given by

$$
\begin{gathered}
\sigma_{d}^{2}(k)=\frac{(6 \sigma U)^{2}}{N+1}\left[2+\cos \left(\frac{2 \pi k}{N+1}\right)\right], \quad k \neq \frac{N+1}{2}, \\
\sigma_{n d}^{2}\left(k, k^{\prime}\right)= \\
\left.\quad \times \cos \left(\frac{\pi k^{\prime}}{N+1}\right)\right], \quad k+k^{\prime} \neq N+1 .
\end{gathered}
$$

From Eq. (16) one can conclude that, in the case of offdiagonal disorder, the motional narrowing effect is also present as it takes place for diagonal disorder, ${ }^{21}$ i.e., both magnitudes in Eq. (16) scale as $(N+1)^{-1}$. We should point out that, in contrast to diagonal disorder, here the magnitudes $\sigma_{d}$ and $\sigma_{n d}$ are functions of the state numbers. Note also that $\sigma_{d}(k)$ goes through its minimum value exactly at the center of the exciton band, i.e., at $k=(N+1) / 2$ and $k=N / 2$ at $N$ taken odd and even, respectively. In fact, we can also assert this with respect to the value of $\sigma_{n d}\left(k, k^{\prime}\right)$ since $k$ and $k^{\prime}$ cannot differ greatly provided the condition $\sigma_{n d}\left(k, k^{\prime}\right) \ll U$.

To conclude this section let us comment on the validity of the motional narrowing effects. Obviously, this is valid only when $\sigma_{n d}<\left|E_{k}-E_{k+1}\right|$. In this case, the excitonic states are not mixed by the perturbation and remain extended over the whole chain. They are essentially mixed for the opposite sign of inequality, reducing their localization lengths. Then, the number of sites within the region of localization $\left(N^{*}\right)$ drives the motional narrowing effect rather than the whole number in the chain $N$. In Refs. 19 and 22 a self-consistent rule for estimation of $N^{*}$ is carried out.

\section{NUMERICAL SIMULATIONS AND DISCUSSIONS}

Further, we will mainly focus our attention on the normalized density of states $\rho(E)$ and on the degree of localization [inverse participation ratio (IPR)] for the states at energy $E$. They are defined, respectively, as follows: ${ }^{17}$ 


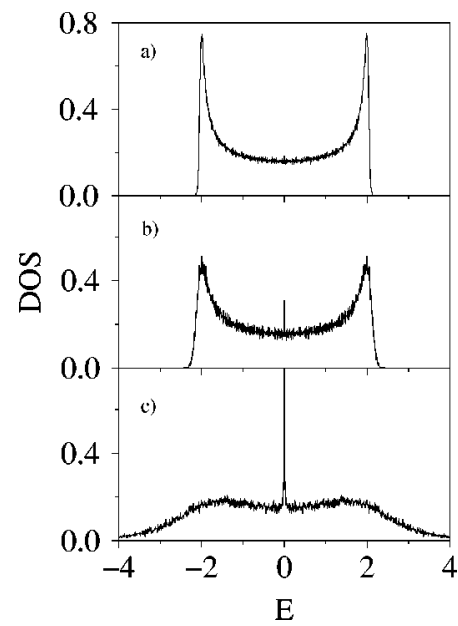

FIG. 1. Density of states in the frame of NN coupling when the lattice size is $N=2500$ and the degree of disorder is (a) $\sigma_{N N}$ $=0.02$, (b) 0.04 , and (c) 0.16 .

$$
\begin{gathered}
\rho(E)=\frac{1}{N}\left\langle\sum_{k} \delta\left(E-E_{k}\right)\right\rangle, \\
\mathcal{L}(E)=\frac{1}{N \rho(E)}\left\langle\sum_{k} \delta\left(E-E_{k}\right)\left(\sum_{n=1}^{N} a_{k n}^{4}\right)\right\rangle,
\end{gathered}
$$

where the angular brackets indicate an average over an ensemble of disordered linear chains and the $a_{k n}$ is the eigenvector of the Hamiltonian (7) corresponding to the eigenvalue $E_{k}$ with $k=1,2,3, \ldots, N$,

$$
\sum_{m=1}^{N} U_{n m} a_{k m}=E_{k} a_{k n} .
$$

The IPR behaves like $1 / N$ for delocalized states spreading uniformly over the entire system on increasing $N$. In particular, the IPR can be exactly computed for the eigenstates of the periodic lattices given in Eq. (9). In doing so we obtain the expected behavior for $N \rightarrow \infty$. On the contrary, localized states exhibit much higher values. In the extreme case, when the exciton is localized at a single site, the IPR becomes unity. Therefore, the scaling analysis of the IPR as a function of the system size provides valuable information about the nature of the excitonic states. We should mention that a complete multifractal analysis, accomplished by studying the scaling of the other moments of the probability distribution, is beyond the scope of this work.

We have solved numerically the eigenvalue problem (18) for different values of disorder, namely, the mean fluctuation of the NN distance, $\sigma_{N N}=\sqrt{2} \sigma$, to study the features of both the DOS and the IPR discussed above. In our numerical treatment $\sigma_{N N}$ ranges from 0 (periodic lattices) up to 0.32 whereas the maximum system size we have considered is $N=2500$. Results comprise averages over 50 realizations of the disorder for each given pair of parameters $N$ and $\sigma_{N N}$.

\section{A. Nearest-neighbor approximation}

Let us comment on the results we have obtained for the NN approximation. Figure 1 shows the DOS for the largest lattice size we have considered $(N=2500)$ and different val-

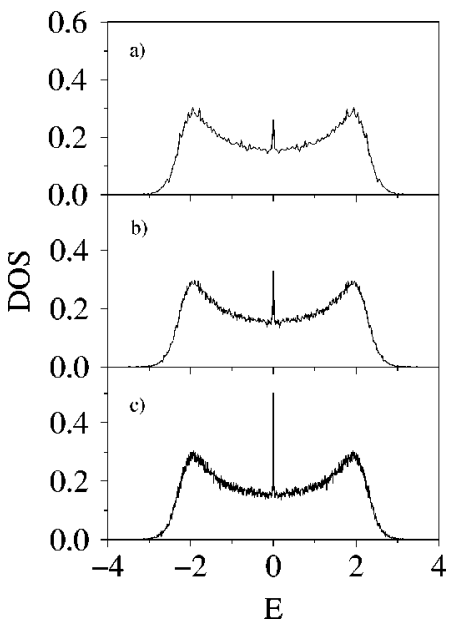

FIG. 2. Density of states in the frame of NN coupling when the degree of disorder is $\sigma_{N N}=0.08$ and the lattice lattice size is (a) $N=1000$, (b) 1500 , and (c) 2500 .

ues of the disorder $\left(\sigma_{N N}=0.02,0.04,0.16\right.$ from top to bottom). We observe that the DOS is symmetric about the center of the band. The singularities at the edge of the exciton band are smeared out on increasing the degree of disorder. Interestingly, a sharp peak in the DOS at the center of the exciton band appears when the degree of disorder exceeds some threshold value ( $\sigma_{N N} \approx 0.03$ for our model parameters). We will discuss this point further later. We have also observed that the percentage of states in the DOS peak increases with the degree of disorder. In addition, the amplitude of the peak rises noticeably with increasing the number of sites in the lattice, as seen in Fig. 2 for $\sigma_{N N}=0.08$.

The IPR presents an overall increase when the degree of disorder increases, meaning that the larger the degree of disorder, the smaller the exciton localization length. This is clearly observed in Fig. 3, where we show the IPR as a function of the exciton energy for the same parameters of Fig. 1. However, the increase of the IPR strongly depends on the energy, being more pronounced close to the center of the band. Simultaneously with the occurrence of the peak of the DOS, a hardly visible dip arises in the IPR at zero energy.

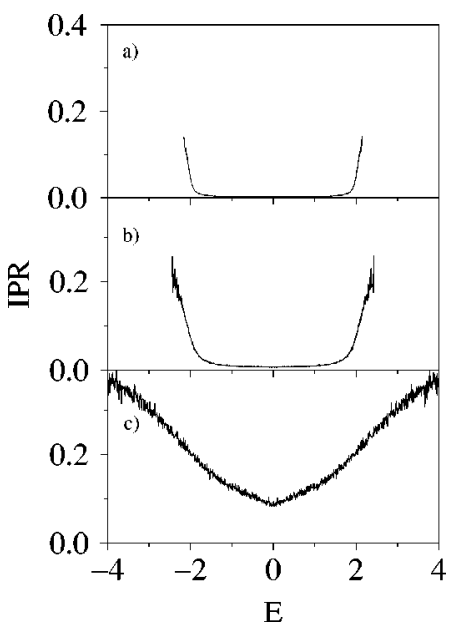

FIG. 3. Inverse participation ratio for the same cases shown in Fig. 1. Notice the overall increase on increasing the degree of disorder. 


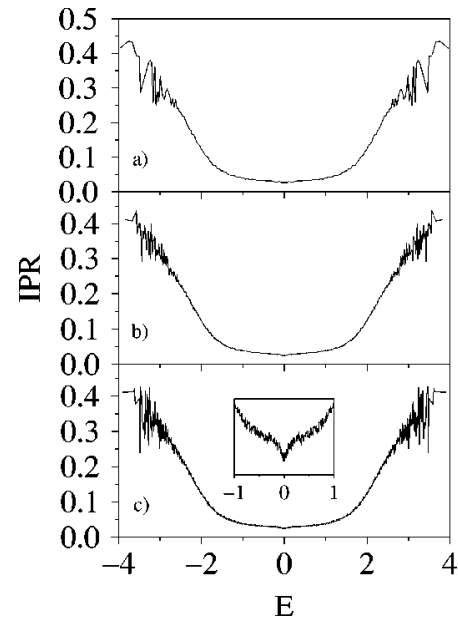

FIG. 4. Inverse participation ratio for the same cases shown in Fig. 2. The inset shows an enlarged view of the center of the band.

This dip is better revealed for larger lattices, as it can be seen in Fig. 4 for the same parameters of Fig. 2.

As mentioned above, the scaling of the IPR with the lattice size may be useful to discern the nature of the eigenstates. The IPR at the center of the band for different values of the degree of disorder is plotted in Fig. 5 as a function of the lattice size. The IPR for periodic lattices scales very accurately as $1 / N$, hence indicating that their eigenstates spread uniformly over the whole lattice. As soon as some amount of disorder is introduced in the system, the IPR follows a power law for small $N$ but tends to a constant value for large $N$, as plotted in Fig. 5. The critical size for which deviation from power fit occurs decreases upon increasing the degree of disorder. The constant value of the IPR for large $N$ increases with the degree of disorder, indicating that the eigenstates at the center of the band actually become more localized.

Summarizing these observations for the NN approximation, we are led to two main conclusions. First, the zeroenergy peak of the DOS really exists. Moreover, as its width shows no dependence on the degree of disorder (at least, when the latter ranges over the interval used in our simulations), we are inclined to identify this peak with a $\delta$-like

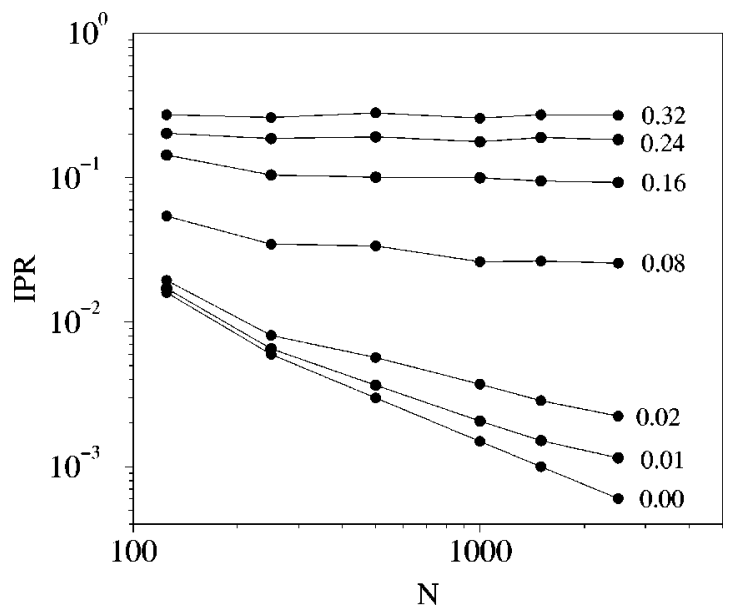

FIG. 5. Scaling of the inverse participation ratio with system size for the eigenstates at the center of the band. Labels indicate the degree of disorder.

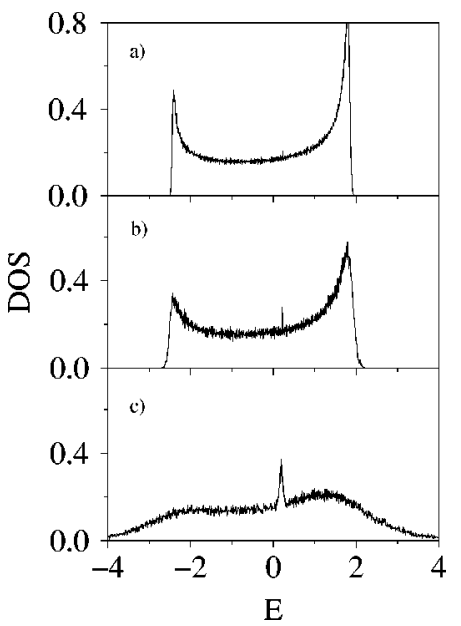

FIG. 6. Density of states in the frame of LR coupling when the lattice size is $N=2500$ and the degree of disorder is (a) $\sigma_{N N}$ $=0.02$, (b) 0.04 , and (c) 0.16 .

singularity rather than to the famous Dyson singularity $\sim 1 /|E| \ln ^{3}|E|$. This singularity was found first for a special distribution of the $\mathrm{NN}$ hopping integral in the form of a generalized Poisson function. ${ }^{10}$ Second, the corresponding eigenstates show no tendency to delocalization with rising lattice size contrary to the opposite statement done in Ref. 9. Moreover, they are not more delocalized than those of energies close to zero.

\section{B. All interactions}

Effects of inclusion of all dipolar interactions in Eq. (18) on the DOS and IPR has been already discussed in Ref. 17. Nevertheless, it has been done only for a fixed value of the chain length $(N=250)$ and the degree of disorder $(\sigma$ $=0.08$ ). Below we present our DOS and IPR numerical data obtained by varying both $N$ and $\sigma$.

Figures 6 and 7 show the results of numerical calculations of both the DOS and the IPR for different values of the degree of disorder and $N=2500$. Here, one can observe the usual changes of both magnitudes as compared to those in the NN approximation: asymmetry and shift of the excitonic

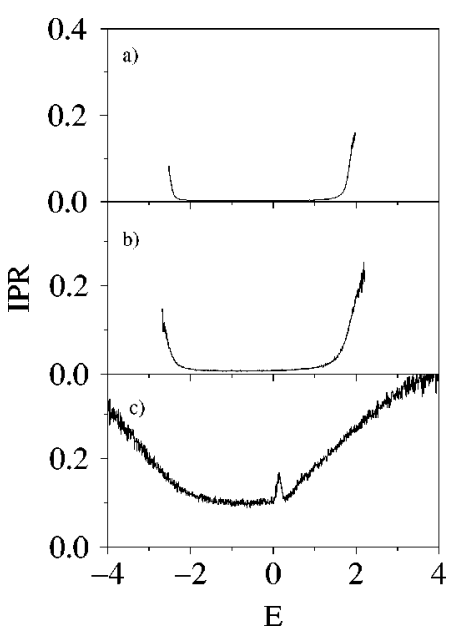

FIG. 7. Inverse participation ratio for the same cases shown in Fig. 6 . 
band edges, both in a good agreement with the analytical results presented in Sec. III A.

In addition, some new features appear, namely, the peak in the DOS has a finite width and is shifted from zero energy to a somewhat higher value $E_{\text {peak }} \approx 0.21 U$ for low degree of disorder, in full correspondence with the results of numerical simulation done in Ref. 17. Higher values of the degree of disorder lead to a smaller energy shift. Further, a peak in the IPR appears at the same energy as the DOS peak, with a finite width as well. The last observation means that the states forming the DOS peak become more localized as compared to those with close energies, in contrast to the case of the NN interaction. This is also confirmed by the scaling of the IPR for $E_{\text {peak }}$ with the system size (not shown here): In all cases we observe higher values of the IPR in comparison with those shown in Fig. 5. Besides that, the trend is similar, that is, the IPR scales as $1 / N$ only for perfect lattices, whereas it tends to a constant value for nonzero degree of disorder.

\section{Discussion}

Now let us discuss the origin of the features of the DOS and of the IPR found in numerical simulations.

\section{1. $N N$ interaction}

Obviously, the zero-energy peak in the DOS might appear when the states at the center of the exciton band become localized, i.e., their localization lengths are reduced to values less than the lattice size. This occurs when the reduced degree of disorder due to motional narrowing, $\sigma_{n d}(0,0)$ $=6 \sigma_{N N} U /(N+1)^{1 / 2}$, exceeds the energy spacing at the center of the band, $\Delta E=2 \pi U /(N+1)$. Equalizing these two magnitudes one obtains an estimation for a threshold of mean fluctuations of the NN distances to observe the peak, $\sigma_{N N}^{t h} \approx U /(N+1)^{1 / 2}$. Thus $\sigma_{N N}^{t h} \approx 0.02$ for $N=2500$, which is in a good agreement with the numerical data of Fig. 1.

With regard to the fact of why this peak appears, we can suggest two explanations that seem to be suitable for the model under consideration. First, as the distribution of disorder we used has long tails then, owing to possible large fluctuations of the $\mathrm{NN}$ distances, strongly interacting dimers can be created whose level splittings noticeably exceed the typical magnitude of the intersite interaction $U$. Consequently, the whole chain is broken into several independent segments in the sense that two adjacent dimers produce a potential well for the exciton, localizing it into the segment bounded by them. As the zero eigenenergy is always present in a segment with odd number of sites, one can expect a peak in the DOS at this energy (a similar explanation of the zeroenergy peak of the DOS was suggested in Ref. 18). The peak amplitude increases with disorder simply because of the rising of the number of segments as the degree of disorder grows. Appearance of such strongly interacting dimers is clearly seen from the fact that the IPR approaches 0.5 at the DOS tails (see Figs. 3 and 4).

Recently, it was demonstrated that the Dyson singularity of the DOS appeared even for a boxlike distribution of disorder. ${ }^{23}$ Then, the explanation above fails due to the absence of large fluctuations of the NN randomness at a low magnitude of the degree of disorder. In such a case, another cause for the occurrence of the zero-energy peak in the DOS can be proposed. As we have already noted in Sec. III B, the first-order correction to the central energy is equal to zero for chains with an odd number of sites and has a minimum of fluctuation in the case of an even number of sites. The zeroenergy peak indicates that the central band eigenenergies are more stable to perturbations than the remaining ones. This certainly will result in a peak of the DOS after averaging over realizations of disorder. It is remarkable that simulations done for a special type of disorder-which has no effect on a certain excitonic level in the sense that the firstorder correction to the energy vanishes-show an analogous peak in the DOS at this energy. ${ }^{24}$ Thus, this empirical rule can serve for inspecting the appearance of peaks in the DOS for the tight-binding Hamiltonian. As the last treatment does not use any specific peculiarities of the NN-randomness distribution, it seems to be suitable for any other distribution. We suppose that, for the model considered in this paper, both mechanisms discussed above contribute to the formation of the zero-energy peak in the DOS.

Concluding this subsection, note that the degree of localization of the central states obtained from the numerical simulation is in a good agreement with the theoretical estimates based on a self-consistent rule proposed in Refs. 19 and 22 (see the first paragraph of the present subsection).

\section{All interactions}

As it was stated in Ref. 17, the energy shift of the DOS peak, $E_{\text {peak }} \approx 0.21 U$, agrees very well with the energy of the central band state in the absence of disorder, Eq. (13). We are also inclined to relate this peculiarity to a state of analogous origin, i.e., similar to $\sin (\pi n / 2)$. This can be demonstrated at least in the perturbative limit. Moreover, exploiting this analogy further, we should assume that the character (having no amplitude on the half of sites) of the mentioned eigenstate has not to be changed dramatically (at least, in average), when passing from the NN model to the exact one, as it is the case for the problem without disorder. ${ }^{19}$

The singularity of the DOS becomes broader with including all dipolar couplings. At least two effects can contribute to this broadening. As it was supposed above, the DOS peak results from the isolated segments of odd number of sites, which, in turn, originate from large fluctuations of the $\mathrm{NN}$ distances. At moderate magnitudes of disorder we are mainly dealing with, the simultaneous strong reduction of the distance between a nearest-neighbor pair and the distances with other neighbors is unlikely. Thus, for the very beginning, one can consider adjacent segments as independent of each other. Then, the eigenenergy of the local (belonging to a certain segment) central band state will fluctuate, owing to fluctuations of the segment lengths [see Eq. (13)], and thus will produce inhomogeneous broadening of the DOS peak. The second probable origin of this effect is the coupling of different isolated segments due to the interaction with far neighbors.

In Ref. 17, the appearance of the IPR peak was explained by an exceptional property of this characteristic with regard to the central band state, $k=(N+1) / 2$, characterized by the wave function $[2 /(N+1)]^{1 / 2} \sin (\pi n / 2)$. Even in the absence of disorder, the IPR defined by Eq. (17b) shows stronger localization of this state $[\mathcal{L}=2 /(N+1)]$ as compared to lo- 
calization of the remaining states $[\mathcal{L}=3 / 2(N+1)] .{ }^{17}$ The authors of Ref. 17 asserted that the IPR peak in the presence of disorder reflected a remnant of this special state in those forming the peak. At the moment, we do not see any other explanation of the origin of this anomaly. If so, a similar feature might be manifested in the NN problem, too. Nevertheless, as follows from our simulations done for the $\mathrm{NN}$ problem, the IPR displays a dip rather than a peak. One reason for such a difference may be the fact that the zeroenergy state is not exponentially localized in the $\mathrm{NN}$ problem (see Sec. II). It results in a larger extension of this state as compared to the others. In principle, such a large extension can compensate the IPR anomaly coming from the special character of the zero-energy state (having no amplitude at all on half of the sites) giving rise to the same value of the IPR at $E=0$ and at surrounding energies.

\section{SUMMARY}

The numerical study of the problem of the zero-energy peak of the DOS for a one-dimensional Frenkel chain with only off-diagonal randomness shows that the peak is really present. In the $\mathrm{NN}$ approximation, it is located at the center of the excitonic band and tends to convert to a singularity as the size of the chain increases. The states belonging to the peak are localized and do not display any tendency to delocalization with increasing chain size. Moreover, the degree of localization (IPR) does not differ very much from that of the surrounding states. The inclusion of couplings due to far neighbors shifts the peak to a slightly higher energy $(\approx 0.21 U)$, while the IPR, in contrast to the NN problem, shows a peak at the same energy.

\section{ACKNOWLEDGMENTS}

Work at Russia was supported by the Russian Foundation for Basic Research (Project No. 95-03-09221). Work at Madrid was supported by CICYT under Project No. MAT950325. V.A.M. thanks Universidad de Salamanca, where this study was started, for hospitality.
${ }^{1}$ P. W. Anderson, Phys. Rev. 109, 1492 (1958).

${ }^{2}$ N. F. Mott and W. D. Twose, Adv. Phys. 10, 107 (1961).

${ }^{3}$ E. H. Lieb and D. C. Mattis, Mathematical Physics in One Dimension (Academic Press, New York, 1966).

${ }^{4}$ S. Alexander, J. Bernasconi, W. R. Schneider, and R. Orbach, Rev. Mod. Phys. 53, 175 (1981).

${ }^{5}$ J. W. Haus and K. W. Kehr, Phys. Rep. 150, 263 (1987).

${ }^{6}$ D. J. Thouless, Phys. Rep. 13, 93 (1974).

${ }^{7}$ D. H. Dunlap, H.-L. Wu, and P. Phillips, Phys. Rev. Lett. 65, 88 (1990).

${ }^{8}$ A. Sánchez, E. Maciá, and F. Domínguez-Adame, Phys. Rev. B 49, 147 (1994).

${ }^{9}$ G. Theodorou and M. H. Cohen, Phys. Rev. B 13, 4597 (1976).

${ }^{10}$ F. Dyson, Phys. Rev. 92, 1331 (1953).

${ }^{11}$ E. N. Economou and M. H. Cohen, Phys. Rev. B 4, 396 (1971).

${ }^{12}$ R. L. Bush, Phys. Rev. B 6, 1182 (1972).

${ }^{13}$ C. T. Papatriantafillou, Phys. Rev. B 7, 5386 (1973).
${ }^{14}$ G. Czycholl, B. Kramer, and A. MacKinnon, Z. Phys. B 43, 5 (1981).

${ }^{15}$ M. Kappus and F. Wegner, Z. Phys. B 45, 15 (1981).

${ }^{16}$ B. Derrida and E. Gardner, J. Phys. (France) 45, 1283 (1984).

${ }^{17}$ H. Fidder, J. Knoester, and D. A. Wiersma, J. Chem. Phys. 95, 7880 (1991).

${ }^{18}$ I. M. Lifshits, S. A. Gredeskul, and L. A. Pastur, Introduction to the Theory of Disordered Systems (Wiley, New York, 1988).

${ }^{19}$ V. Malyshev and P. Moreno, Phys. Rev. B 51, 14587 (1995).

${ }^{20}$ I. S. Gradshtein and I. M. Ryzhik, Table of Integrals and Products (Academic Press, New York, 1980).

${ }^{21}$ E. W. Knapp, Chem. Phys. 85, 73 (1984).

${ }^{22}$ V. A. Malyshev, Opt. Spektrosk. 71, 873 (1991) [Opt. Spectrosc. 71, 505 (1991)]; J. Lumin. 55, 225 (1993).

${ }^{23}$ J. Mertsching, Phys. Status Solidi B 174, 129 (1992).

${ }^{24}$ G. G. Kozlov (unpublished). 